\title{
Minimising treatment-associated risks in systemic cancer therapy
}

\author{
Ulrich Jaehde · Andrea Liekweg $\cdot$ Sven Simons • \\ Martina Westfeld
}

Received: 6 March 2007 / Accepted: 21 August 2007/Published online: 29 September 2007

(C) Springer Science+Business Media B.V. 2007

\begin{abstract}
Aim of the review To review the consequences of drug-related problems (DRP) in systemic cancer therapy and identify specific contributions of the pharmacist to minimise treatment-associated risks. Method Searches in PubMed, Embase and the Cochrane Library were conducted. Bibliographies of retrieved articles were examined for additional references. Only papers in English between 1980 and 2007 were included. Results In systemic cancer therapy there is an enormous potential for DRP due to the high toxicity and the complexity of most therapeutic regimens. The most frequently reported DRP can be classified into adverse effects, drug-drug interactions, medication errors, and non-adherence. Pharmacists have enhanced efforts to assure quality and safety in systemic cancer therapy together with other health care providers. In consequence, oncology pharmacy has evolved as a novel specialist discipline. The endeavour to merge and coordinate individual activities and services of the pharmacist has led to pharmaceutical care concepts which aim at offering novel solutions to the various DRP. Conclusion Pharmaceutical care for cancer patients should be developed within research projects and integrated into disease management programs in order to ensure broad implementation.
\end{abstract}

Keywords Cancer Chemotherapy ·

Drug-related problems · Medication errors · Oncology ·

Pharmaceutical care

U. Jaehde $(\bowtie) \cdot$ A. Liekweg $\cdot$ S. Simons $\cdot$ M. Westfeld Institute of Pharmacy, Department of Clinical Pharmacy, University of Bonn, Rheinische Friedrich-Wilhelms-Universitaet Bonn, An der Immenburg 4, 53121 Bonn, Germany e-mail: u.jaehde@uni-bonn.de

\section{Impact of findings on practice}

- There is an enormous potential for drug-related problems in cancer therapy

- The most frequently reported drug-related problems in systemic cancer therapy are adverse effects, drug-drug interactions, medication errors, and non-adherence

- Pharmacists can contribute substantially to risk minimisation in systemic cancer therapy by adding specific drug-related knowledge to the treatment team and offering patient-related services

- Pharmaceutical care for cancer patients offers novel solutions to the various drug-related problems and should be further developed in research projects

\section{Introduction}

In systemic cancer therapy, drug regimens are administered following established protocols which have been carefully evaluated in clinical trials. The administration of supportive medication is not as standardised as with antineoplastic therapy. Major components of the supportive therapy are selected by the general practitioners or the patients themselves rather than by the oncologist. Furthermore, patients tend to see more than one physician involved in the cancer care process as well as alternative practitioners. Patients are also exposed to a tremendous variety of drugs which are available to the customer without prescription (overthe-counter, OTC).

The more complex drug therapy is, the higher the risk of experiencing drug-related problems (DRP) such as adverse effects, interactions, medication errors, and non-adherence. Drug-related problems in cancer chemotherapy can have 
severe consequences originating from the high toxicity and narrow therapeutic range of anticancer drugs. The tragic case of Betsy Lehman who died from an overdose of cyclophosphamide at the Dana Faber Cancer Center in Boston demonstrated this risk painfully [1]. It must be the goal of all health care providers to minimise treatmentassociated risks as much as possible.

Over the last few decades the pharmacy profession has experienced a change from traditional drug-oriented toward patient-oriented services. In oncology, pharmacists have established central services for compounding cytotoxic drugs and started to offer industry-independent drug information for physicians and patients as well as therapeutic drug monitoring for critical dose drugs, e.g. aminoglycosides in neutropenic patients. As the specialist knowledge of pharmacists in this field has increased continuously "oncology pharmacy" has evolved into a new pharmaceutical discipline with its own curriculum. In 1995 the International Society for Oncology Pharmacy Practitioners (ISOPP) was founded. The aim of the society is "to determine the optimal medical treatment for cancer patients, thereby improving their quality of life". Currently, the concept of pharmaceutical care is being adapted to the needs of the cancer patient as a further step to optimising individual drug therapy [2].

The intention of this review is to summarise possible solutions for DRP in oncology focussing on the specific tasks of the pharmacist.

\section{Aim of the review}

It was the aim of this article to review and analyse the most frequent DRP and their consequences in systemic cancer therapy. Moreover, specific contributions of the pharmacist to minimise treatment-associated risks should be identified.

\section{Method}

Searches in PubMed, Embase and the Cochrane Library were conducted. Database terms included DRP, adverse effects, drug-drug interactions, medication errors, adherence, compliance, pharmaceutical care in connection with oncology or cancer. Bibliographies of retrieved articles were examined for additional references. Only papers in English between 1980 and 2007 were included.

\section{Results}

Various DRP can occur in the treatment path of systemic cancer therapy (Table 1). In a recently published study
Table 1 Drug-related problems can originate from several steps of the treatment path

\begin{tabular}{ll}
\hline Treatment path & Potential hazard \\
\hline $\begin{array}{l}\text { Prescription } \\
\text { Ordering }\end{array}$ & Adverse effects \\
$\begin{array}{l}\text { Compounding } \\
\text { Storage } \\
\quad \text { Administration } \\
\text { Monitoring }\end{array}$ & $\begin{array}{l}\text { Interactions } \\
\text { Medication errors }\end{array}$ \\
\hline
\end{tabular}

conducted on an oncology ward of a Swedish hospital 114 DRP in 58 patients were identified indicating their high incidence in cancer patients [3]. In this section, major problems related to adverse effects, drug-drug interactions, medication errors, and non-adherence are reviewed separately.

\section{Adverse effects}

According to the World Health Organization (WHO) an adverse drug reaction (ADR) is defined as a response to a drug which is noxious and unintended, and which occurs at doses normally used or tested in man for prophylaxis, diagnosis or therapy of disease. In cancer chemotherapy such ADRs are strongly connected to the treatment itself. Because of the fact that most cytotoxic agents cannot distinguish between normal and neoplastic cells, most ADRs seem to be unavoidable. They are often accepted not only by patients but also by health care providers.

In order to illustrate the most common ADRs in oncology patients in a cancer centre in Australia were observed and the incidence as well as the predictability, preventability, and severity of the occurred ADRs were assessed. Among the ten most common ADRs constipation ranked first but was connected directly to the use of opioids rather than to cytotoxic chemotherapy. Nausea and vomiting, fatigue, alopecia, drowsiness and myelosuppression ranked second to sixth. Of these ADRs $88 \%$ were predictable and about $50 \%$ even probably preventable, because of inadequate use of preventative measures [4].

As well as the incidence of ADRs in modern chemotherapy the patients' perceptions of the impact of these ADRs on well being and quality of life are increasingly taken into account. Patient perceptions have changed markedly over the last two decades. Whereas in 1983 physical symptoms such as nausea, vomiting and hair loss were most troubling from the patients' point of view, in 2002 psychosocial complaints ranked among the top ten symptoms, with the complaint "affects my family or partner" rated as the most severe ADR. Alopecia and fatigue ranked second and third place $[5,6]$. 
As a consequence, the management of ADRs has become a focus of clinical research and new drugs such as aprepitant, a neurokinin-1-receptor antagonist for the prevention of nausea and vomiting, were developed [7]. Despite these new therapeutic options the minimisation of toxic effects of chemotherapy still seems to be a challenging task.

The first and most important step to a better management of ADRs is the formulation and implementation of evidence-based clinical practice guidelines for the different symptoms in a multidisciplinary approach. Several studies have shown a positive effect of guidelines for antiemetic prophylaxis and therapy on both clinical and economic outcomes [8-11]. The most important influencing factors seem to be the appropriate use of $5-\mathrm{HT}_{3}$ receptor antagonists and the application of oral drug formulations. Nevertheless, altering the usage patterns of physicians to comply with evidence-based guidelines seems to be difficult. Methods such as the use of computerised decision support systems and educational outreach mechanisms appear to be most effective particularly when used in combination [12]. The pharmacist can support and promote the adherence to guidelines [13].

A second step to a better management of ADRs is the implementation of standardised chemotherapy order forms that also contain supportive care medication. By using standardised order forms a more appropriate prescribing of antiemetics based on the level of emetogenicity of administered chemotherapy and thus a reduction in drug expenditure can be achieved [14]. Such order forms should be elaborated following a multidisciplinary approach including physicians, pharmacists and nurses.

\section{Drug-drug interactions}

The large number of prescribed drugs administered to cancer patients leads to a high potential for drug-drug interactions. As indicated previously many cancer patients also use over-the-counter medication as well as alternative and complementary treatment options. Frequently, cancer patients suffer from concomitant chronic diseases that require the intake of other drugs, further increasing the risk of interactions. This problem is often underestimated in oncology as highlighted in several review articles [15-18]. Moreover, drug-food, drug-disease and drug-diagnostics interactions have to be considered that are, however, beyond the scope of this article.

Drug-drug interactions must be avoided as they can lead to overdosing or underdosing of anticancer drugs with the consequence of toxicity or a loss of effectiveness, respectively. However, not all published interactions are also clinically relevant. A rational and safe drug therapy includes a check for potential drug-drug interactions based on the individual medication and a decision whether and how identified drug-drug interactions have to be considered or not.

Knowledge of the mechanisms of interactions is crucial to assess the clinical relevance of an interaction. Drug-drug interactions are usually classified as pharmaceutical, pharmacokinetic or pharmacodynamic interactions (Table 2). The risk of interactions can differ substantially between individual drugs, even within the same class of drugs. This can be considered when selecting a particular drug for an individual patient.

For some drugs with a high potential for interactions, therapeutic drug monitoring can be useful to detect and control interactions. The advantage of this approach is that the dosage of the drug of risk can be adapted to the measured individual plasma concentrations. Thus the treatment of both interacting drugs may be continued. Examples are anticonvulsant, antidepressant and antifungal drugs which are often administered to cancer patients as supportive medication [19].

In general, it is almost impossible for the physician to keep in mind all of the interaction mechanisms and potential consequences for the individual patient. Regular interaction checks by the pharmacist may solve this problem as he is the only health care professional who may have an overview of the drugs prescribed by various physicians and the medication taken by the patient on his own initiative. The avoidance of drug-drug interactions should be regarded as a multidisciplinary task [20].

The basis for each interaction check is to take the medication history of each patient and to update it regularly. Nowadays software tools are available facilitating rapid interaction checks and providing information on the mechanism and clinical relevance of an interaction. Each hospital and community pharmacy should have access to at least one of these tools.

Table 2 Classification of drug-drug interactions

\section{Pharmaceutical interactions}

Mostly physical or chemical incompatibilities, e.g. chemical reactions or precipitations due to drug admixtures.

\section{Pharmacokinetic interactions}

Occur at the level of drug absorption, distribution, excretion, and metabolism. Frequently, the cytochrome P450 metabolising enzyme system and drug transporters such as P-glycoprotein are involved in such interactions.

\section{Pharmacodynamic interactions}

Directly related to the desired or undesired drug effects, e.g. the antitumoral effect or drug-associated toxicity. 


\section{Medication errors}

In addition to the DRP described above, there are numerous risks for the occurrence of medication errors along the therapeutic path in oncology. A medication error is defined as any preventable event that may cause or lead to inappropriate medication use or patient harm, while the medication is in the control of the health care professional, patient, or consumer [21]. In 2000 the American Institute of Medicine published their report "To err is human: Building a Safer Health System" which analysed flaws in the health system and offered suggestions for its improvement [22]. Based on the results of a study conducted in Utah and Colorado in 1992 an incidence of about $3 \%$ adverse events in hospitalised patients was found, of which the majority of the non-operative events were adverse drug events (ADE) [23]. The authors concluded that iatrogenic injury continues to be a significant public health problem. Due to the narrow therapeutic range of many anticancer drugs, the impact of an ADE is more serious (and at worst lethal) compared to other systemically administered drugs. Antineoplastic agents were found to be the second most common group of drugs which caused lethal medication errors [24]. As well as quality and safety issues, the reduction of medication errors has a financial effect.

The first source of medication errors is the prescription and ordering process: the wrong protocol may be chosen, the cumulative dose may be mixed up with the single dose, the route of administration may not be clearly indicated or misinterpretation of the physicians' handwriting may lead to errors. A study carried out in a cytotoxic preparation unit in a French hospital revealed in only a 6 month period more than 300 medication errors out of 1,262 prescriptions for 285 patients. Most errors $(>70 \%)$ were found to be simple physicochemical incompatibilities, e.g. incompatibility of the drug with the used matrix. However, also overand underdosage as well as wrong medications were identified [25]. The authors conclude that most of these errors could possibly be avoided by a computerised prescription network. Computer-assisted solutions enable physicians to order the medication electronically which is nowadays one way to reduce the incidence of those errors. Mekhjian et al. evaluated the benefits of physician order entry systems and found that transcription errors could be reduced and speed in the ordering process could be improved. Even the duration of the stay in hospital could be reduced by the system [26]. Particularly in oncology, the computerised physician order entry (CPOE) has obvious advantages. A recent study has shown that CPOE in electronic medical records improves completeness of the medical record compared to paper charts independent of regimen complexity [27]. Prepared protocols facilitate correct ordering and double checking by the programme itself and by the pharmacist responsible. In addition, supportive medication, such as hydration or the antiemetic or antiallergic medication can be automatically proposed depending on the selected regimen. Moreover, the risk of misinterpretation of handwriting is eliminated. Recently, a CPOE system with integrated clinical decision support has been implemented in Ontario (Canada). This system, for example, alerts the clinician when maximum cumulative doses are reached, calculates body surface area, and estimates the creatinine clearance of the patient [28]. With all the advantages of these systems it has to be taken into account that new risks may arise from those new processes such as information errors due to the lack of clearness on the monitor [29].

The next step after ordering, the compounding of cytotoxic drugs also holds various risks, especially when performed on the ward. The implementation of central cytotoxic preparation units in pharmacy departments in the late 1980s has been one of the first measures to standardise the process in order to increase safety. Nevertheless, of 30,819 preparations surveyed in a study on incidence and risk factors of preparation errors 140 were found to be defective $(0.45 \%)$ [30]. Less than half of those cases were classified as major errors including wrong dosage, wrong labelling or the use of incompatible diluents. Although the incidence is fairly low it must still be the aim to reduce this further by analysing the risk factors such as workload. Centralisation itself offers many more options for safety improvement. Standardised pharmaceutical validation leads to an increased interception of medication errors in antineoplastic treatment. This can be explained by the improved knowledge of the pharmacists involved in the validation process. In consequence of a standardised pharmaceutical validation process in the hematologyoncology department of a 550-bed university hospital the number of detected medication errors that did not reach the patients could be increased by $41 \%$ (from 14.08 medication errors/1,000 patient days to 19.83 medication errors/ 1,000 patient days) in the second year after the introduction of pharmacists in the multidisciplinary oncology team [31]. Using their expertise, pharmacists can facilitate protocol development and adherence, dose verifications and education of other health care practitioners [32].

Finally, storage and administration errors of cytotoxic drugs form a group of major medication errors with serious consequences for the patient. Therefore, it is mandatory that the assigned staff are well trained in all aspects of chemotherapy storage and administration. For many drugs, special storage conditions, e.g. refrigeration or protection from light, have to be considered. Detailed knowledge of the administered drugs is also very important in order to educate the patient and to react appropriately when 
incidents such as extravasations occur. In case of extravasation the necessary measures have to be initiated. Different drugs require different measures which the oncologically trained staff needs to know. An extravasation kit including essential items for first aid must be made available on site [33]. Incorrect or delayed treatment can cause serious damage to the patient, resulting in even the loss of a limb.

Various surveys have been undertaken in order to find solutions for the prevention of medication errors. The nonpunitive reporting of errors is a necessary requirement to be able to detect, analyse and consequently minimise the incidence and severity of medication errors in oncology. Safety measures with error reporting and analysis have been developed and implemented in order to improve the entire system [34]. The most effective improvements of treatment performance have been achieved by multidisciplinary system approaches integrating physicians, pharmacists and nurses [2, 32, 34-36].

\section{Non-adherence}

Adherence (or compliance) is generally defined as the extent to which a person's behaviour, taking medication, following a diet, and/or executing lifestyle changes, corresponds with agreed recommendations from a health care provider [37]. Non-adherence strongly compromises the success of a patient's therapy, results in additional, potentially unnecessary, diagnostic and therapeutic procedures and thus generates further costs and possibly health problems as a consequence of the treatments themselves. It is important to note that the traditional concept that patients are solely responsible for taking their medication (and therefore non-adherence is a patient-driven problem) is misleading. The WHO understands adherence as a phenomenon consisting of five dimensions (Table 3). The magnitude of this problem is further emphasised by the fact that adherence rates for many long-term drug therapies range from only 40 to $78 \%[38,39]$.
It is a difficult task to assess patient adherence reliably. Patient self-reports, rates of prescription refills, patient diaries or pill counts as the sole basis for the measurement of adherence have been shown to be inadequate [38-40]. It has been demonstrated that these methods overestimate the degree to which patients adhere to their tamoxifen regimen [40]. The use of microelectronic adherence monitoring (Medication Event Monitoring System, MEMS ${ }^{\mathrm{TM}}$, Fig. 1) provides a valuable estimate of the timing of events and insights into patients' behaviour in taking medication. However, to prove actual intake of the medication, additional plasma concentration monitoring would be necessary. To obtain an optimal measurement of patient adherence, a combination of several methods is suggested [38].

Studies conducted in various disease areas have shown that a wide variety of methods to improve adherence is available. A recently published review classifies these methods into four general categories: patient education, improved dosage, increased hours when clinics are open, and improved communication between health care providers and patients [38]. All four categories require a collaborative approach including all members of the multidisciplinary health care team. Due to their position in the chain of health care providers, this requires the contribution of pharmacists, not only in the hospital setting but also in community pharmacies [41]. It has been shown that no single intervention strategy appears consistently more effective than another. Successful methods are complex and comprehensive as well as labour-intensive [38, 42]. The Cochrane Review on Interventions to Enhance Medication Adherence concludes that there is no evidence that low adherence can be "cured" and hence efforts to improve adherence must be maintained for as long as the treatment is needed [43].

Cancer patients seem to benefit especially from interventions towards an optimised adherence, resulting in improved outcomes [42]. Research investigating adherence in oncology settings has been mostly focussed on palliative care and supportive medication because chemotherapy has mainly been administered intravenously in hospitals or

Table 3 The five dimensions of adherence and examples of associated factors (adapted from [34])

\begin{tabular}{lllll}
\hline Social/economic factors & $\begin{array}{l}\text { Health care team/system-related } \\
\text { factors }\end{array}$ & Condition-related factors & Therapy-related factors & Patient-related factors \\
\hline Economic status & Patient-provider relationship & Severity of symptoms & Complexity of regimen & Anxiety about side effects \\
Cultural beliefs & Education of providers & Level of disability & Treatment duration & Patients' motivation \\
Illiteracy & Capacity of system & Rate of progression & Changes in treatment & Patients' expectations \\
Age & Duration of consultations & Co-morbidities & Side effects & Forgetfulness \\
$\begin{array}{l}\text { Distance from treatment } \\
\text { center }\end{array}$ & Medication distribution system & Availability of effective & Previous treatment & Patients' knowledge \\
freatments & failures & about illness \\
\hline
\end{tabular}




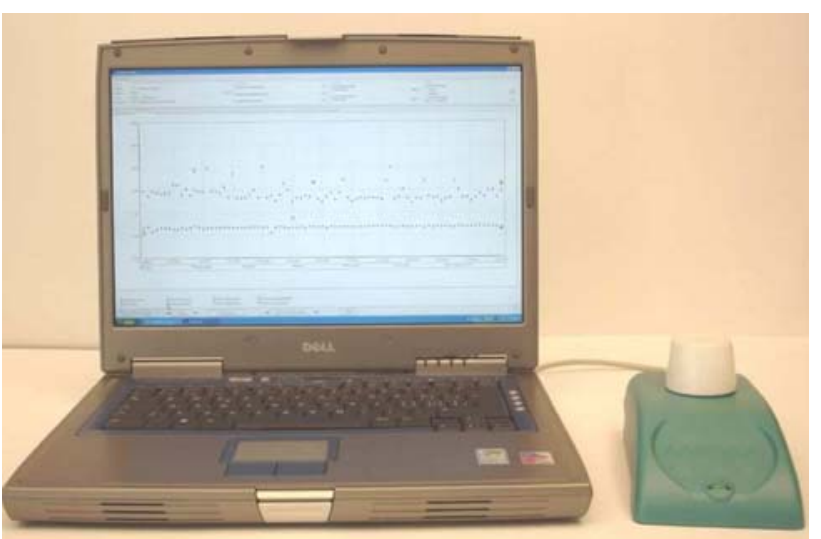

Fig. 1 Medication event monitoring system, $\operatorname{MEMS}^{\mathrm{TM}}$ : The pill bottle cap contains a micro-electronic circuit that registers when the bottle is opened. This data may then be transferred to a computer via a reading device

clinics. However, with the increasing availability and importance of oral anticancer drugs such as capecitabine, etoposide, vinorelbine, erlotinib and sorafenib, patients increasingly take over the responsibility for the correct administration of their prescribed therapy. Although cancer patients might be more adherent than other patient groups due to a high level of motivation, the slightest non-adherence can endanger the therapeutic goals [39].

Only few studies have been published investigating the level of adherence of cancer patients. In a cohort of outpatients receiving chemotherapy for haematologic malignancies adherence to the allopurinol and prednisolone prescription was measured based on plasma concentrations. It was shown that control patients without intervention were adherent only $17 \%$ of the time with allopurinol and $27 \%$ of the time with prednisone. Three specially developed intervention packages including education and home visits resulted in increased adherence rates of $44-48 \%$ and $33-38 \%$ of the time for allopurinol and prednisone, respectively [44]. Another study assessed adherence in patients with breast cancer receiving oral cyclophosphamide and found that $43 \%$ of the patient population met criteria for non-adherence according to both behavioural and dosage definitions [45]. In a recently published study in a cohort of 2816 women adherence to tamoxifen treatment was investigated using prescription refill data: $22.1 \%$ of the patients exhibited a discontinuation of treatment (nonpersistence) within 1 year [46]. An open controlled trial is currently undertaken at the University of Bonn investigating the impact of intensified pharmaceutical care provision on the adherence of patients receiving oral capecitabine using the MEMS ${ }^{\mathrm{TM}}$-technology. Pharmacists' interventions include the extensive provision of drug information to the patients and health care professionals, routine checks for drug interactions, preparation of written drug intake plans for patients, regular pharmacist consultations and structured documentation of the pharmaceutical care process [47].

\section{Discussion}

The recognition of the described risks of the individual patient associated with complex drug therapies has led to the development of a conceptual framework for an advanced pharmacy practice philosophy. The concept of pharmaceutical care was introduced as a further development of the pharmaceutical profession gaining acceptance in Europe and worldwide [48, 49]. The American Society of Health System Pharmacists set up guidelines for standardised pharmaceutical care to ensure that pharmacists practicing pharmaceutical care work to the same standard [50].

Patients with complex drug regimens and/or chronic diseases and those who frequently need to be hospitalised might benefit from pharmaceutical care in particular. These criteria apply to many cancer patients. Within the pharmaceutical care process the application of agreed therapeutic algorithms can be assured on the individual basis. The adherence of the patient can be improved by patient education before and during the treatment cycles combined with patient counselling regarding drug therapy, adverse effects and complementary treatment options.

The London oncology pharmacy group introduced guidelines for pharmaceutical care of cancer patients which not only include the actual 'pharmaceutical care' as such, but standardise the clinical pharmacy activities, dispensing, updating therapeutic policies, cytotoxic reconstitution, drug information, clinical trials, and the oncology training of pharmacists [51]. A group of British experts has drawn up a policy framework for commissioning cancer services. They suggest the establishment of structures which support the seamless care of cancer patients in the community setting in a network of all parties in order to make use of the respective specialist knowledge [52]. Accordingly, information flow at discharge from hospital to the community should be optimised utilising pharmaceutical care plans to ensure the efficient distribution of medication to the patient is not interrupted.

Although there are some reports on the implementation of pharmaceutical care for cancer patients there is still little scientific evidence on the feasibility of pharmaceutical care and its actual benefit to the patient. In Canada, projects have been carried out which suggest to implement suitable outcome parameters to evaluate the impact of pharmaceutical services in oncology [53]. These have stimulated a wide discussion in Canadian health care politics regarding the necessity of the services offered. In Scotland, research 
is concentrating on the documentation and thereby standardisation of the provision of pharmaceutical care to cancer patients [54]. In Germany, various research projects are being carried out which investigate the feasibility and benefit of pharmaceutical care for patients with different indications. Patients with breast and colorectal cancer as well as patients receiving oral chemotherapy are the focus of projects at the University of Bonn. Another project is being carried out in Hamburg developing the provision of pharmaceutical care to lung cancer patients [2].

In terms of cancer many disciplines contribute to the care process. Thus, cross-profession and cross-sector cooperation is crucial in order to improve information flow. Over the last few years disease management programs (DMPs) have increasingly been introduced to cover the whole care process which begins with the early diagnosis of the disease. They aim at providing the optimal medical care by the implementation of evidence-based guidelines. Pharmaceutical care concepts seem to have the potential to support the goals of DMPs and could be easily integrated in programmes for cancer patients. However, it is necessary to document the impact of pharmaceutical care on patient outcomes in order to comply with the demand for transparency.

\section{Conclusions}

Systemic cancer therapy is particularly complex and hence associated with multiple risks for the patient as described above. Many of these risks are preventable by specific measures which can be taken by a particular health care provider or the patient. The pharmacist with his central position relating to drug dispensing and utilisation can contribute substantially by adding specific drug-related knowledge and offering patient-related services.

Pharmaceutical care is designed as a framework that integrates individual contributions of the pharmacist into the entire therapeutic path. Model projects are urgently needed to assess the clinical, humanistic and economic outcome of patient-oriented pharmaceutical services. The integration of pharmaceutical care into disease management programmes might facilitate efficient collaboration between all health care professionals and hence improve effectiveness and safety of systemic cancer therapy.

Acknowledgement We gratefully acknowledge the financial support of the "Förderinitiative Pharmazeutische Betreuung e.V." Conflicts of interests The authors declare that they have no conflicts of interests.

\section{References}

1. Knox RA. Doctor's orders killed cancer patient. Boston Globe 1995;23:1 Metro/Region.
2. Liekweg A, Westfeld M, Jaehde U. From oncology pharmacy to pharmaceutical care: new contributions to multidisciplinary cancer care. Support Care Cancer 2004;12:73-9.

3. Bremberg ER, Hising C, Nylen U, Ehrsson H, Eksborg S. An evaluation of pharmacist contribution to an oncology ward in a Swedish hospital. J Oncol Pharm Pract 2006;12:75-81.

4. Lau PM, Stewart K, Dooley M. The ten most common adverse drug reactions (ADRs) in oncology patients: do they matter to you? Support Care Cancer 2004;12:626-33.

5. Coates A, Abraham S, Kaye SB, Sowerbutts T, Frewin C, Fox $\mathrm{RM}$, et al. On the receiving end-patient perception of the sideeffects of cancer chemotherapy. Eur J Cancer Clin Oncol 1983;19:203-8.

6. Carelle N, Piotto E, Bellanger A, Germanaud J, Thuillier A, Khayat D. Changing patient perceptions of the side effects of cancer chemotherapy. Cancer 2002;95:155-63.

7. Sharma R, Tobin P, Clarke SJ. Management of chemotherapyinduced nausea, vomiting, oral mucositis, and diarrhoea. Lancet Oncol 2005;6:93-102.

8. Dranitsaris G, Leung P, Warr D. Implementing evidence based antiemetic guidelines in the oncology setting: results of a 4-month prospective intervention study. Support Care Cancer 2001;9:611-8.

9. Rough SS, Carro GW. Developing and implementing oral 5-HT3 receptor antagonist guidelines: a multidisciplinary process. Pharm Pract Manag Q 1998;18:48-58.

10. Berard CM, Mahoney CD. Cost-reducing treatment algorithms for antineoplastic drug-induced nausea and vomiting. Am J Health Syst Pharm 1995;52:1879-85.

11. Engstrom C, Hernandez I, Haywood J, Lilenbaum R. The efficacy and cost effectiveness of new antiemetic guidelines. Oncol Nurs Forum 1999;26:1453-8.

12. Bero LA, Grilli R, Grimshaw JM, Harvey E, Oxman AD, Thoson MA. Closing the gap between research and practice: an overview of systematic reviews of interventions to promote the implementation of research findings. The cochrane effective practice and organization of care review group. BMJ 1998;317:465-8.

13. Dranitsaris G, Warr D, Puodziunas A. A randomized trial of the effects of pharmacist intervention on the cost of antiemetic therapy with ondansetron. Support Care Cancer 1995;3:183-9.

14. Sano HS, Waddell JA, Soimando DA, Doulaveris P, Myhand R. Study of the effect of standardized chemotherapy order forms on prescribing errors and anti-emetic cost. J Oncol Pharm Pract 2005;11:21-30.

15. Beijnen JH, Schellens JH. Drug interactions in oncology. Lancet Oncol 2004;5:489-96.

16. Larn MSH, Ignoffo RF. A guide to clinically relevant drug interactions in oncology. J Oncol Pharm Pract 2003;9:45-85.

17. McLeod HL. Clinically relevant drug-drug interactions in oncology. Br J Clin Pharmacol 1998;45:539-44.

18. Blower P, de Wit R, Goodin S, Aapro M. Drug-drug interactions in oncology: why are they important and can they be minimized? Crit Rev Oncol Hematol 2005;55:117-42.

19. Joerger M, Schellens JH, Beijnen JH. Therapeutic drug monitoring of non-anticancer drugs in cancer patients. Methods Find Exp Clin Pharmacol 2004;26:531-45.

20. Garabedian-Ruffalo SM, Syrja-Farber M, Lanius PM, Plucinski A. Monitoring of drug-drug and drug-food interactions. Am J Hosp Pharm 1988;45:1530-34.

21. American Society of Consultant Pharmacists. Guidelines on preventing medication errors in pharmacies and long-term care facilities through reporting and evaluation. http://www.ascp.com/ advocacy/briefing/patientsafety.cfm (accessed 06 March 2007).

22. Kohn LT, Corrigan JM, Donaldson MS. To err is human: building a safer health system. Washington, DC: National Academy Press; 2000. 
23. Thomas EJ, Studdert DM, Burstin HR, Orav EJ, Zeena T, Williams EJ, et al. Incidence and types of adverse events and negligent care in Utah and Colorado. Med Care 2000;38:261-71.

24. Phillips J, Beam S, Brinker A, Holquist C, Honig P, Lee LY, et al. Retrospective analysis of mortalities associated with medication errors. Am J Health Syst Pharm 2001;58:1835-41.

25. Slama C, Jerome J, Jacquot C, Bonan B. Prescription errors with cytotoxic drugs and the inadequacy of existing classifications. Pharm World Sci 2005;27:339-43.

26. Mekhjian HS, Kumar RR, Kuehn L, Bentley TD, Teater P, Thomas A, et al. Immediate benefits realized following implementation of physician order entry at an academic medical center. J Am Med Inform Assoc 2002;9:529-39.

27. Harshberger CA, Brockstein B, Carro G, Jiang W, Spath W, Lawton J. Evaluation of outcomes before and after electronic medical record (EMR) and computerized physician order entry (CPOE) system implementation in an outpatient oncology setting [ASCO Annual Meeting Proceedings]. J Clin Oncol 2007;25(18S): 17058.

28. Greenberg A, Kramer S, Welch V, O’Sullivan E, Hall S. Cancer Care Ontario's computerized physician order entry system: a province-wide patient safety innovation. Healthc Q 2006;9(Spec Issue):108-13.

29. Koppel R, Metlay JP, Cohen A, Abaluck B, Localio AR, Kimmel $\mathrm{SE}$, et al. Role of computerized physician order entry systems in facilitating medication errors. JAMA 2005;293:1197-1203.

30. Limat S, Drouhin JP, Demesmay K, Tissot E, Jacquet M, Woronoff-Lemsi MC. Incidence and risk factors of preparation errors in a centralized cytotoxic preparation unit. Pharm World Sci 2001;23:102-6.

31. Fabia AS, Rodrigo EC, Mari AA, Cubells DA, Torres NVJ. Pharmaceutical validation as a process of improving the quality of antineoplastic treatment. J Oncol Pharm Pract 2005;11:45-50.

32. Goldspiel BR, DeChristoforo R, Daniels CE. A continuousimprovement approach for reducing the number of chemotherapy-related medication errors. Am J Health Syst Pharm 2000;57(Suppl 4):S4-S9.

33. Mader I, Fürst-Weger PR, Mader RM, Semenitz EI, Terkola R, Wassertheurer SM. Extravasation of cytotoxic agents: compendium for prevention and management. Heidelberg: Springer; 2003.

34. Womer RB, Tracy E, Soo-Hoo W, Bickert B, DiTaranto S, Barnsteiner JH. Multidisciplinary systems approach to chemotherapy safety: rebuilding processes and holding the gains. J Clin Oncol 2002;20:4705-12.

35. France DJ, Miles P, Cartwright J, Patel N, Ford C, Edens C, et al. A chemotherapy incident reporting and improvement system. Jt Comm J Qual Saf 2003;29:171-80.

36. Mueller T. Typical medication errors in oncology: analysis and prevention strategies. Onkologie 2003;26:539-44.
37. WHO. Adherence to long-term therapies-evidence for action. Geneva: World Health Organization; 2003.

38. Osterberg L, Blaschke T. Adherence to medication. N Engl J Med 2005;353:487-97.

39. Partridge AH, Avorn J, Wang PS, Winer EP. Adherence to therapy with oral antineoplastic agents. J Natl Cancer Inst 2002;94:652-61.

40. Waterhouse DM, Calzone KA, Mele C, Brenner DE. Adherence to oral tamoxifen: a comparison of patient self-report, pill counts, and microelectronic monitoring. J Clin Oncol 1993;11:1189-97.

41. Van Wijk BL, Klungel OH, Heerdink ER, de Boer A. Effectiveness of interventions by community pharmacists to improve patient adherence to chronic medication: a systematic review. Ann Pharmacother 2005;39:319-28.

42. Roter DL, Hall JA, Merisca R, Nordstrom B, Cretin D, Svarstad B. Effectiveness of interventions to improve patient compliance: a meta-analysis. Med Care 1998;36:1138-61.

43. Haynes R, Yao X, Degani A, Kripalani S, Garg A, McDonald HP. Interventions to enhance medication adherence. Cochrane Database Syst Rev 2005;CD000011.

44. Levine AM, Richardson JL, Marks G, Chan K, Graham J, Selser $\mathrm{JN}$, et al. Compliance with oral drug therapy in patients with hematologic malignancy. J Clin Oncol 1987;5:1469-76.

45. Lebovits AH, Strain JJ, Schleifer SJ, Tanaka JS, Bhardwaj S, Messe MR. Patient noncompliance with self-administered chemotherapy. Cancer 1990;65:17-22.

46. Barron TI, Connolly RM, Bennett K, Feely J, Kennedy MJ. Early discontinuation of tamoxifen. Cancer 2007;109:832-839.

47. Simons S, Roth S, Arjukese A, Braun M, Ko Y, Kuhn W, et al. Measuring compliance in patients receiving oral capecitabine. Onkologie 2006;29(Suppl.3):205.

48. Hepler CD, Strand LM. Opportunities and responsibilities in pharmaceutical care. Am J Hosp Pharm 1990;47:533-43.

49. Van Mil JW, Schulz M, Tromp TF. Pharmaceutical care, European developments in concepts, implementation, teaching, and research: a review. Pharm World Sci 2004;26:303-11.

50. ASHP guidelines on a standardized method for pharmaceutical care. American Society of Health-System Pharmacists. Am J Health Syst Pharm 1996;53:1713-6.

51. Hoare D, Beer C. Guidelines for the pharmaceutical care of cancer patients. Pharm J 1995;255:841-2.

52. Working Party Report. Pharmaceutical care of cancer patients in the community. Pharm J 1997;258:54-8.

53. Broadfield L. Pharmaceutical care in oncology pharmacy practice: A method for using outcome indicators. J Oncol Pharm Pract 1995;1:9-14.

54. Macintyre J, Dalrymple H, MacLean F, Lannigan N, Hudson SA. Development of a system for reporting pharmaceutical care issues in cancer patients receiving chemotherapy. Pharm $\mathrm{J}$ 2003;271:266-7. 